\title{
TUNGSTEN CARBIDE NANOPOWDER SYNTHESIS UNDER THE EXPOSURE OF 24 GHZ GYROTRON RADIATION ON THE NANOCOMPOSITE OF THE W-C SYSTEM OBTAINED IN A THERMAL PLASMA
}

\author{
A. Vodopyanov ${ }^{1,2}$, A. Samokhin ${ }^{2}$, N. Alekseev ${ }^{2}$, M. Sinayskiy ${ }^{2}$, A. Sorokin ${ }^{1}$, S. Sintsov $^{1}$ \\ ${ }^{1}$ Institute of Applied Physics RAS, 46 Ul'yanov st., Nizhny Novgorod, Russia \\ ${ }^{2}$ Baikov Institute of Metallurgy and Material Science RAS, 49 Leninskiy av., Moscow, Russia \\ avod@yandex.ru
}

Keywords: microwave, gyrotron, tungsten carbide, nanoparticles, synthesis microwave

\section{Introduction}

Nanoscale tungsten carbide WC powders are of practical interest for creating nanostructured hard alloys with enhanced physical and mechanical characteristics, wearresistant nanostructured coatings, electro-catalysts in fuel cells, metal melt modifiers [1-8]. An efficient method for producing tungsten carbide nanopowders is a plasma-chemical synthesis of a multicomponent $\mathrm{W}-\mathrm{C}$ powder nanocomposite with its subsequent heat treatment. It results in the formation of tungsten carbide WC [2, 9-11]. Experimental studies have shown the possibility of producing tungsten carbide WC nanopowder using this method. Heat treatment in the electric furnace at a temperature of $1100-1200^{\circ} \mathrm{C}$ for a few hours is necessary for the complete conversion of the nanocomposite into the target product. The heating is accompanied by an increase of nanoparticles in size.

Currently, much attention is paid to the heating of reagents by microwaves to conduct solid-phase chemical reactions [12-19]. Microwave heating provides energy deposition in the volume of the processed material, this significantly increases the heating rate compared to traditional heating in furnaces, where energy is transferred from the surface to the volume of material. Numerous studies have shown that the use of microwave heating for chemical reactions in solids makes it possible to increase the rate by order of magnitude, to increase the yield of target products and to reduce the impurities. The use of microwave heating with a radiation frequency of $2.45 \mathrm{GHz}$ for the synthesis of tungsten carbide WC from powder mixtures of tungsten or tungsten trioxide with carbon leads to a reduction in the reaction time [20-27]. It also provides the possibility to obtain the target product in the form of a nano-sized powder using tungsten precursors having a particle size in the nanometer range.

In the most cases, studies of chemical syntheses using microwave heating were carried out in an electromagnetic field with a frequency of $2.45 \mathrm{GHz}$, but it is noted that an increase in the frequency of the waves may result in additional effects of material synthesis $[13,19$, 28].

The purpose of the research was an experimental study of the possibility of obtaining tungsten carbide WC from a powder multicomponent nanocomposite system W-C, obtained by plasma-chemical synthesis when processed in the microwave field with two different frequencies: $2.45 \mathrm{GHz}$ and $24 \mathrm{GHz}$.

\section{Experimental Technique}

The W-C nanopowder processing experiments were conducted on two available microwave sources: the gyrotron complex with a nominal output power of $7 \mathrm{~kW}$ in $\mathrm{CW}$ mode with radiation frequency of $24 \mathrm{GHz}$ and a magnetron with a radiation frequency of $2.45 \mathrm{GHz}$ and an average power of $0.8 \mathrm{~kW}$. The use of two sources of microwave radiation made it possible to investigate the dependence of the properties of the treated nanopowder $\mathrm{W}-\mathrm{C}$ on the wavelength of the heating field while other conditions being equal. 
The gyrotron complex can be structurally divided into a treatment chamber with a microwave radiation focusing system and a microwave system. Fig. 1a shows the experimental setup. The gyrotron radiation with a frequency of $24 \mathrm{GHz}$ in is introduced by a circular waveguide with a diameter of $32.6 \mathrm{~mm}$ through a sealed window into the processing chamber. The microwave radiation converted from the TE11 mode of a circular waveguide into a Gaussian beam. Then it was focused by a parabolic 90 -degree mirror ( 1 in Fig. 1A). The Gaussian beam was focused on the surface of the processed nanopowder placed in a quartz crucible ( 2 in Fig. 1A). The amount of nanopowder loaded in a quartz crucible and processed in one experiment was about $10 \mathrm{~g}$. There is a region of the focal waist at a distance of $32 \mathrm{~cm}$ from the center of the parabolic mirror. The cross-sectional area of the beam was $3 \mathrm{~cm}^{2}$. The power of the gyrotron radiation varied from 100 to 400 watts in the experiments described. The sample surface temperature was 1050-1200 C. The processing chamber was sealed; all the flanges entrances on its body are standard CF vacuum fittings. The flange entrance ( 3 in Fig. 1A) was made according to the CF250 standard and has a vacuum quick access door with a viewing port covered with a metal grid. Visual control of the sample heating processes and the measurement of the surface temperature were performed using a RAYTEK Marathon MR1S two-color pyrometer through the viewing port. Also, this door used to load and unload the crucible with nanopowder. Samples of nanosized powder $\mathrm{W}-\mathrm{C}$ were processed in a stream of nitrogen at atmospheric pressure.

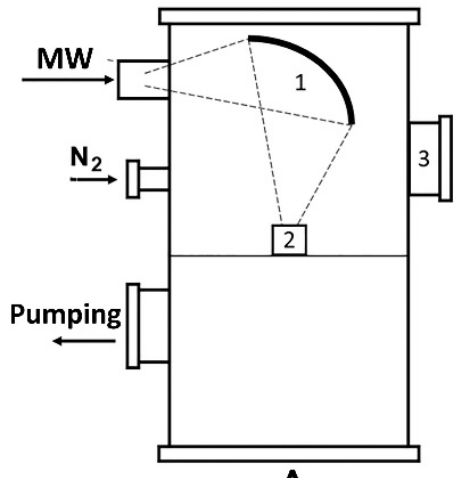

A

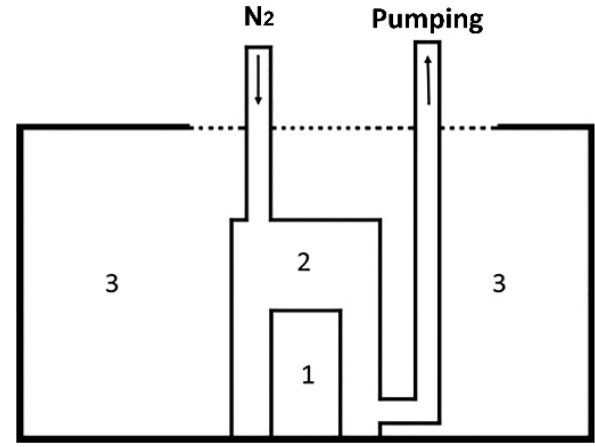

B

Fig. 1. Schemes of experimental installations.

Fig. 1B shows the setup for heating a W-C nanopowder with microwave radiation with a frequency of $2.45 \mathrm{GHz}$. The processing chamber housing is a commercial microwave oven (3 in Fig. 1B). There was a quartz reactor (2 in Fig. 1B) inside the microwave oven which isolates the ceramic crucible (1 in Fig. 1B) with the nanopowder being treated from the external air atmosphere.

The quartz reactor has 2 entrances for nitrogen purge and pumping out. The pressure inside the reactor was maintained slightly excessive relative to the atmosphere to ensure continuous nitrogen purging.

The powders obtained in the experiments were analyzed using following techniques: phase composition using an X-ray diffractometer Rigaku Ultima IV; total carbon content using a LECO CS-600 analyzer; the specific surface of the powders using a Micromeritics TriStar 3000 specific surface analyzer; morphology and particle size of powders using a Scios FEI electron microscope. 


\section{The starting materials}

A nanopowder of the W-C system was used for processing in the microwave field. This nanopowder was obtained by the interaction of tungsten oxide $\mathrm{WO}_{3}$ powder with methane in a stream of hydrogen-nitrogen plasma generated in an electric arc plasma torch [9]. The initial nanopowder consists of the carbide phases $\mathrm{WC}_{1-\mathrm{x}}$ and $\mathrm{W}_{2} \mathrm{C}$ mainly with the presence of metallic W and free carbon. The total carbon content in the nanopowder was $6.3 \mathrm{wt} . \%$. The specific surface area was $22 \mathrm{~m}^{2} / \mathrm{g}$. The nanopowder consists of highly aggregated particles with sizes less than $100 \mathrm{~nm}$ (see Fig. 2). The nanopowder contains particles of micron size range as impurities, consisting of tungsten oxide and metal tungsten, the mass content of such particles was about $10 \%$. Processing of nanopowders was carried out in a nitrogen environment. The experiments high purity $(99,9999 \%)$ compressed nitrogen and evaporated liquid nitrogen was used.

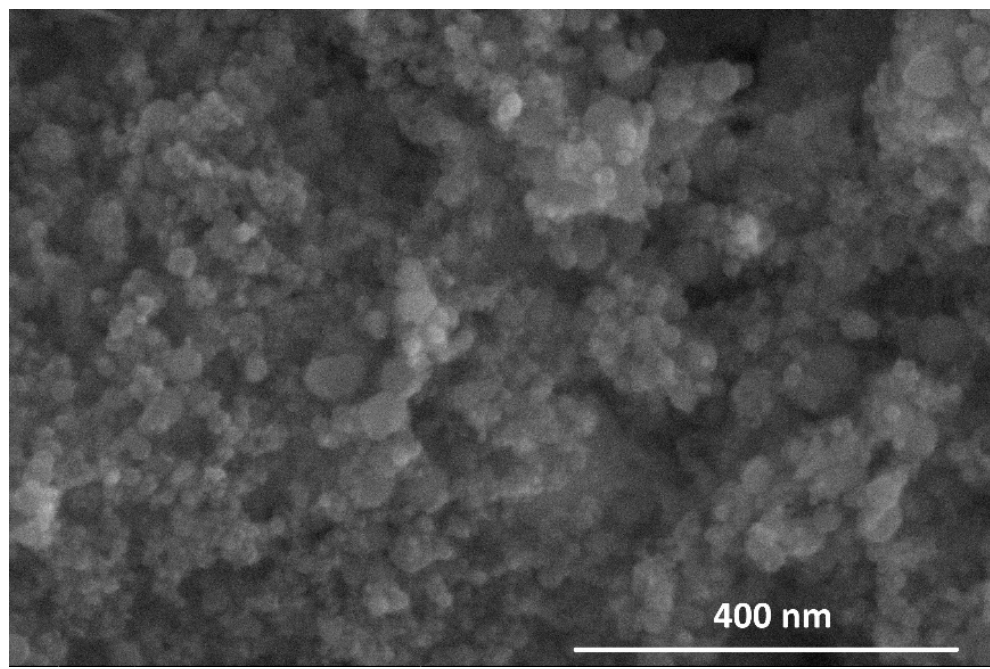

Fig. 2. Microphoto of initial nanopowder.

\section{Results and Discussion}

One of the main factors determining the course of solid-phase chemical reactions during direct heating of reagents in a microwave field is the absorption efficiency of reagents and reaction products, which determine its temperature. Experiments have shown that the original nanopowder absorbs well the used microwave radiation with both frequencies of $2.45 \mathrm{GHz}$ and $24 \mathrm{GHz}$. A sample of the original nanopowder with a mass of 10-12 $\mathrm{g}$ was heated to a temperature of $1100^{\circ} \mathrm{C}$ for exposure time on the order of several seconds. The temperature variation during the process was $50-70^{\circ} \mathrm{C}$ with a significant change in the phase composition of the nanopowder as a result of the transformation of $\mathrm{WC}_{1-\mathrm{x}}->\mathrm{WC}$. This fact indirectly indicates the proximity of the absorption properties of these carbides at the frequencies of microwave radiation used in the experiments.

Results of experiments performed at a constant microwave power of $260 \mathrm{~W}$ with a frequency of $24 \mathrm{GHz}$ shows that chemical transformation of the original nanopowder occur from the first minutes of the microwave processing and is characterized by a decrease of the tungsten carbide $\mathrm{WC}_{1-\mathrm{x}}$ content and an increase of the $\mathrm{WC}$ carbide (see Fig. 3). The highest transformation rate was observed in the first minutes. During the subsequent time (up to 12.5 minutes) the residual content of carbide $\mathrm{WC}_{1-\mathrm{x}}$ practically does not change with a certain 
increase in the content of carbide WC. The maximum processing time was $12.5 \mathrm{~min}$, however, during this time, the complete conversion of the components of the original nanopowder to tungsten carbide WC was not achieved, which may be due to a decrease in the total carbon content in the nanopowder during microwave processing (see Fig. 4). Tungsten carbide WC is a stoichiometric compound and has no homogeneity region; therefore, lower carbides are formed with a lower carbon content with a decrease in the total carbon content in the reaction system. The decrease in total carbon content could be due to its chemical ablation as a result of the interaction of free carbon and tungsten carbides with oxygen impurities present in the nitrogen used to purge the powder treatment chamber. The experiment showed the effect of the nitrogen type used for purging the composition of the nanopowder treated. The parameters were the following: the frequency of microwaves 24 $\mathrm{GHz}$, heating power was $260 \mathrm{~W}$, treating time $5 \mathrm{~min}$. The sample treated in a stream of evaporated liquid nitrogen characterized by a higher conversion of $\mathrm{WC}_{1-\mathrm{x}}$ to $\mathrm{WC}$. The presence of oxygen provides the possibility to transfer carbon required for the formation of $\mathrm{WC}$ from $\mathrm{WC}_{1-\mathrm{x}}$ through the gas phase through the reactions $\mathrm{O}_{2}+\mathrm{Cs}_{\mathrm{S}}-\mathrm{CO}, \mathrm{CO}->\mathrm{Cs}_{\mathrm{S}}+$ $\mathrm{CO}_{2}$.

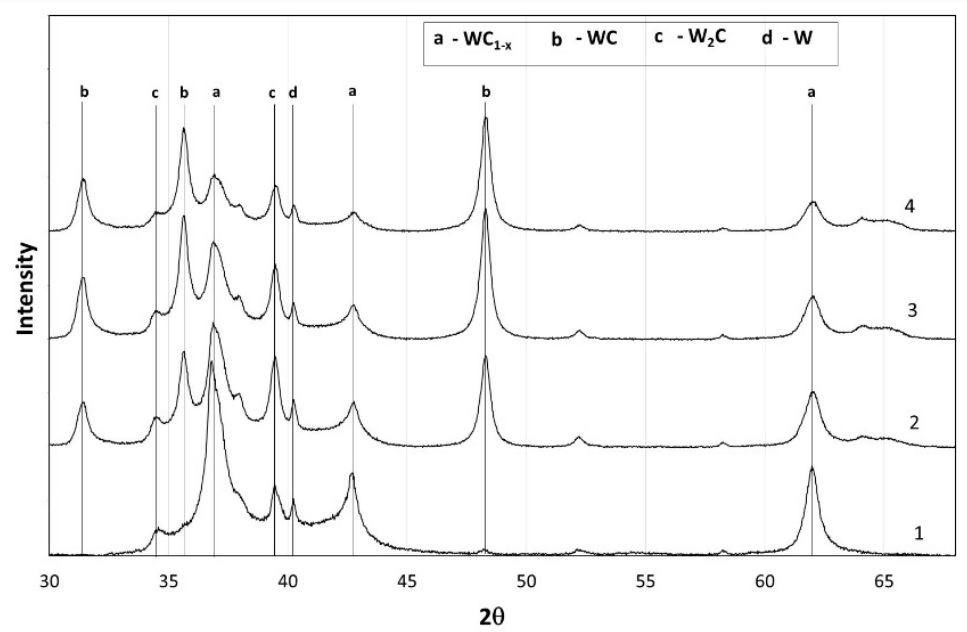

Fig. 3. X-ray diffraction pattern of nanopowders after the microwave treatment $(24 \mathrm{GHz}$, $260 \mathrm{~W}$ ) during the various time. 1 - initial, 2 - 2 min., 3 - 5 min., $4-12.5 \mathrm{~min}$. 


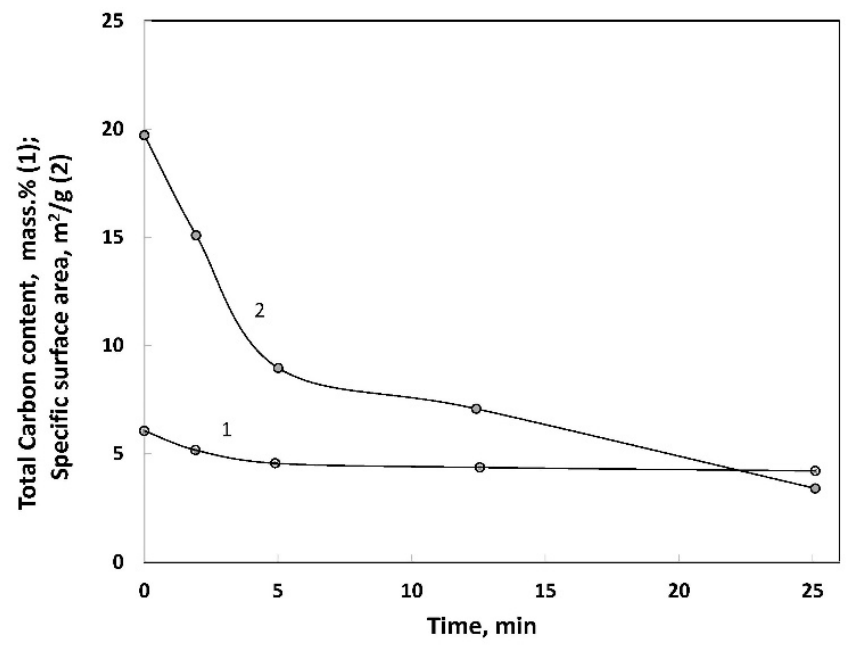

Fig. 4. Change in time for total carbon content and for the specific surface in nanopowders at the microwave treatment $(24 \mathrm{GHz}, 260 \mathrm{~W})$.

The specific surface of the powder being processed decreases when tungsten carbide $\mathrm{WC}$ is formed under microwave heating with a frequency of $24 \mathrm{GHz}$ (see Fig. 4) and the specific surface area decreases from $22 \mathrm{~m}^{2} / \mathrm{g}$ to $12 \mathrm{~m}^{2} / \mathrm{g}$ in $25 \mathrm{~min}$ of processing, while the particle size remains in the corresponding nanoscale range (see Fig. 5A).

An increase in the power of microwaves with a frequency of $24 \mathrm{GHz}$ with a constant processing time of 5 min leads to an increase in the output of tungsten carbide WC (see Fig. $6)$.

The phase composition and the morphology of the obtained products are mostly the same when processing samples in the microwaves with a frequency of $2.45 \mathrm{GHz}$ with a power of $800 \mathrm{~W}$ and when processing samples in the microwaves with a frequency of 24 GHz (see Fig. 5b and Fig. 7). A significant change in the phase composition (transformation of $\mathrm{WC}_{1-\mathrm{x}}->\mathrm{WC}$ ) also occurs during the first minutes, and from 3 to 8 minutes the WC content increases much slowly (see Fig. 7). There is a decrease in the total carbon content and a decrease in the specific surface area of the powder during processing when used microwaves with a frequency of $2.45 \mathrm{GHz}$ likely when used microwaves with a frequency of $24 \mathrm{GHz}$.
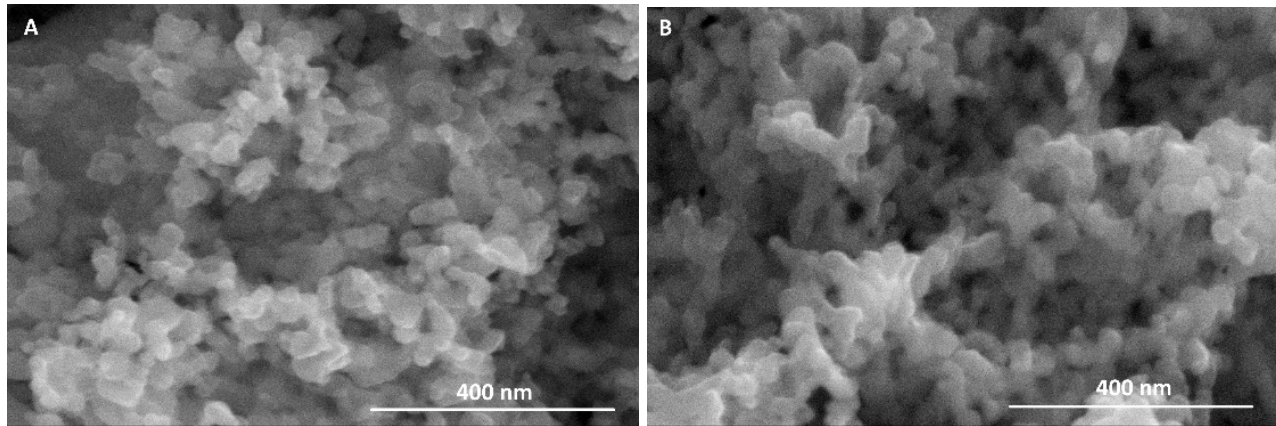

Fig. 5. Microphoto of nanopowders after the microwave treatment. A - $24 \mathrm{GHz}, 370 \mathrm{~W}, 5$ min. $\mathrm{B}-2.45 \mathrm{GHz}, 800 \mathrm{~W}, 5 \mathrm{~min}$. 


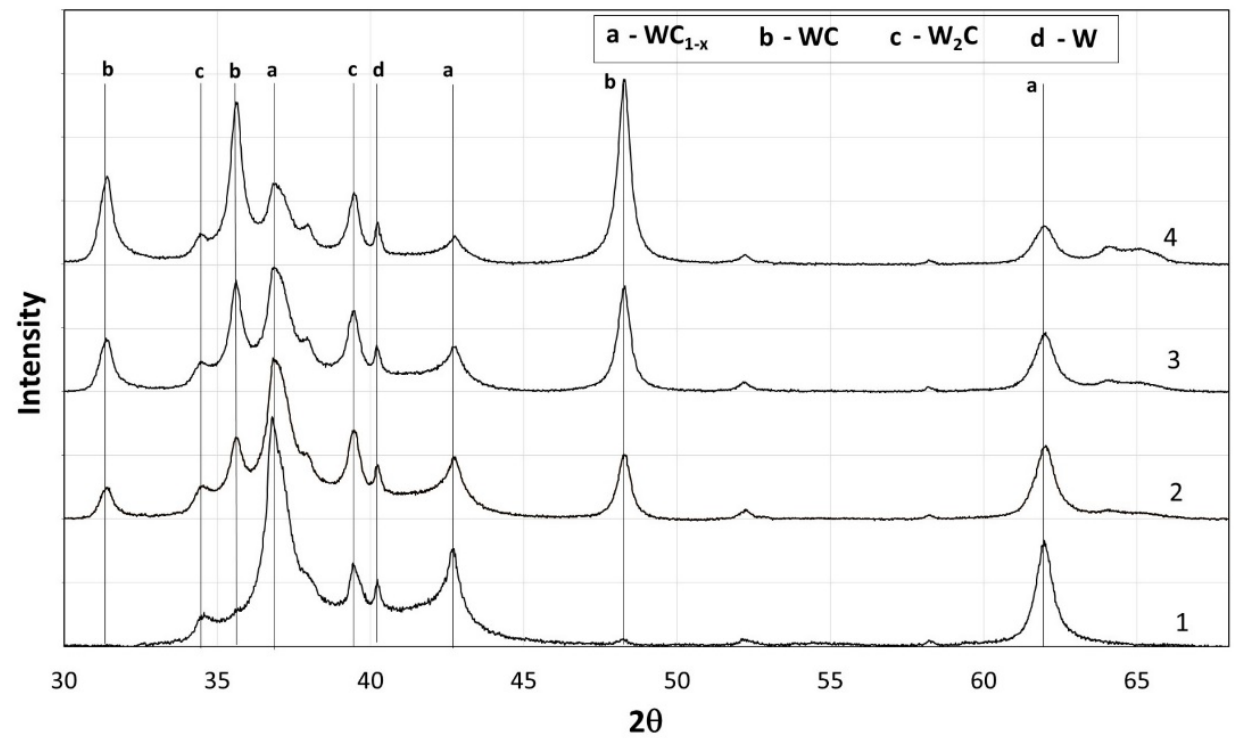

Fig. 6. X-ray diffraction pattern of nanopowders after the microwave treatment $(24 \mathrm{GHz}, 5$ min.) at various power. 1 - initial, $2-260 \mathrm{~W}, 3-310 \mathrm{~W}, 4-370 \mathrm{~W}$

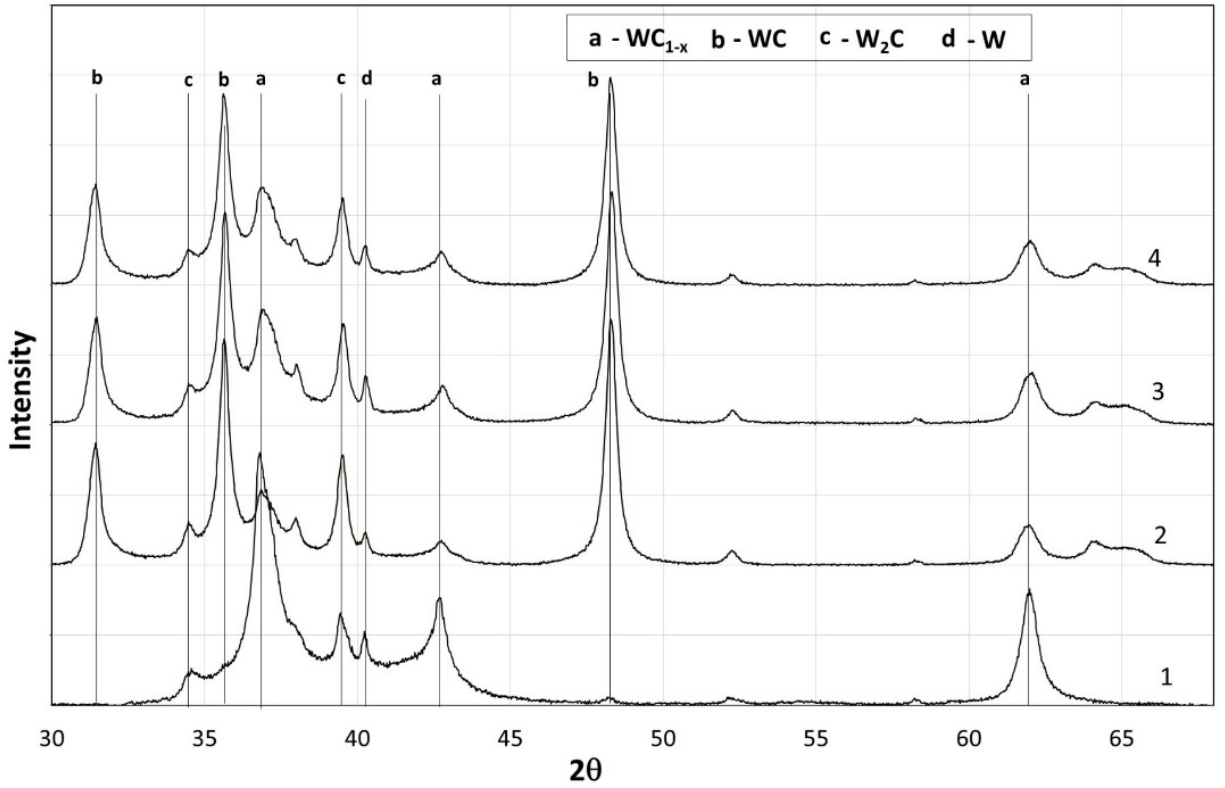

Fig. 7. X-ray diffraction pattern of nanopowders after the microwave treatment $(2.45 \mathrm{GHz}$, $800 \mathrm{~W}$ ) during the various time. 1 - initial, $2-3 \mathrm{~min} ., 3-5 \mathrm{~min} ., 4-8 \mathrm{~min}$.

\section{Conclusion}

Experimental studies performed have demonstrated the possibility of direct heat treatment of multicomponent nanopowders of the W-C system obtained by plasma-chemical synthesis, when exposed to a microwave field with frequencies of 2.45 and $24 \mathrm{GHz}$. The formation of nanoscale particles of tungsten carbide WC occurs when microwaves used for 
processing while there is a decrease in the value of the specific surface area of the nanopowder. The process of chemical and phase transformation is affected by the composition of the gas medium, which components can provide gas-phase carbon transfer.

Subsequent studies will determine the possibility to achieve $100 \%$ yield of WC while maintaining the particle size of the powder in the nanometer range.

The work was carried out within the framework of the Program \#14 "Physical chemistry of adsorption phenomena and actinide nanoparticles" of the Presidium of the Russian Academy of Sciences.

\section{References}

1. Panov V.S. Metal Ceramics, 2015, 53(11-12), 643-654.

2. Z. Zak Fang, Xu Wang, Taegong Ryu, et al.. Int. J. of Refr. Met. and Hard Mater., 2009, 27(2), 288-299.

3. Kurlov A.S., Gusev A.I. Tungsten Carbides. Struct., Prop., and Appl. in Hardmetals, Springer 2013, 242 p.

4. Kalita, V.I., Komlev, D.I., Yashin, V.B., et al., Fiz. Khim. Obrab. Mater. (in rus), 2011, 5, 32-43.

5. Sokolov G.N., Zorin I.V., Artemev A.A., et al.. Inorg. Mater.: Appl. Research, 2015, 6(3), 240-248.

6. Linnik A.A., Pankratov A.S., Kobernik N.V. Izv. Vissh. Ucheb. Zaved. Mashinostroenie (in rus), 2013, 6, 66-71.

7. Nikiforov A., Petrushina I., Christensen E., Alexeev N.V., Samokhin A.V., Bjerrum N.. Int. J. of Hydrogen Energy, 2012, 37 (24), 18591-18597.

8. S. Emina, C. Altinkayab, A. Semercib, H. Okuyucub, A. Yildizb, P. Stefanovic.. Appl. Catalysis B: Environmental, 2018, 236, 147-153.

9. Samokhin A. V., Alekseev N. V., Kornev S. A. Plasma Chem. and Plasma Proc., 2013, 33(3), 605-616.

10. Taegong Ryu, H.Y. Sohn, Kyu Sup Hwang, Zhigang Z. Fang. J. Mater. Sci., 2008, 43, 5185-5192.

11. T. Ryu, H. Y. Sohn, K. S. Hwang, Zh. Z. Fang. J. Am. Ceram. Soc., 2009, 92(3), 655-660.

12. Kitchen H.J., Vallance S.R., Kennedy J.L., et al. Chem. Rev. 2014, 114, 1170-1206.

13. Y. Makino. ISIJ Int., Vol. 47 (2007), No. 4, pp. 539-544.

14. K. J. Rao, B. Vaidhyanathan, M. Ganguli, P. A. Ramakrishnan. Chem. of Mater., 1999, 11 (4), 882-895.

15. Kappe C.O. Am Lab. 2001, 33, 13-19.

16. Dąbrowska S. Chudoba T., Wojnarowicz J .Łojkowski W. A Review. Crystals 2018, 8, 379-405.

17. Leonelli C., Lojkowski W. Chem. Today, 2007, 25 (3), 34-38.

18. Leonelli C., Komarneni S. Inorganics, 2015, 3, 388-391.

19. Imam M. A., Lewis D., Bruce R. W., Fliflet A. W., Kurihara L.K. Mater. Sc. Forum, 2003, 426-432, 4111-4116.

20. K. Essaki, E. J. Rees, G.T. Burstein. J. Am. Ceramic Soc.2009, 93 (3), $692-695$.

21. A. Karimzadeh, B. Arman, H. Masoud, S.Email, M. Sh. Bafghi, K. Yanagisawa. Int. J. of Miner. Metall. and Mater., 2017, 24 (2), 202-207.

22. Nikolaenko I., Kedin N., Shveikin G., Polyakov E. Int. J. of Mater. Res. 2014, 105 (3), 314-317.

23. J. L. Lu, Z. H. Li, S. P. Jiang, et al. J.of Power Sources, 2012, 202, 56- 62.

24. Vallance S.R., Kitchen H.J., Ritter C., et al. Green Chem., 2012, 14, 2184-2192. 
25. Behnami A.K., Hoseinpur A., Sakaki M., et al. Int. J. of Miner. Metall., and Mater., 2017, 24 (2), 202-207.

26. Wu A.-H., Tang J.-C., Ye N., et al. Mater. Sc. and Enginer. Powd. Metall. 2014, 19(6), 862-866.

27. Vallance S.R., Kingman S., and Gregory D.H. Adv. Mater. 2007, 19, 138-142.

28. Bykov Yu.V., Rybakov K.I., Semenov V.E.. J. Phys. D: Appl. Phys., 2001, 34, 5575. 